\title{
UNCERTAINTY IN DEPTH EVALUATION OF PARTIALLY CONDUCTIVE CRACKS FROM EDDY CURRENT TESTING SIGNALS
}

The paper deals with eddy current non-destructive evaluation of partially conductive cracks. Especially, uncertainty of a depth estimation of such cracks from eddy current testing signals is considered here. A plate specimen having the electromagnetic parameters of a stainless steel SUS316L is inspected in this study by numerical means. A crack with variable depth, width and conductivity is positioned in the middle of the plate. One of the most utilized eddy current probes so called pancake probe is employed for the inspection. Response signals of the crack with different parameters are numerically calculated and further analysed. Presented results clearly demonstrate that the uncertainty of a crack depth estimation considerably increases when conductive cracks are evaluated comparing to non-conductive cracks.

\section{Introduction}

Many structures require periodical inspection to keep safety, reliability as well as quality of various processes. Recent trends in maintenance are embracing the so-called damage tolerance approach, wherein an element is actively used up to a certain point, beyond which the structural integrity of a structure could be affected. Replacement is thus performed at the end of the service life of the element, which helps in lowering running costs of the whole system. Degradation detection of the construction material must be accompanied by interpretation of the measured data in order to estimate the extent of the degradation and predict future development thereof.

The damage tolerance approach includes four phases - detection, evaluation, analysis and prediction. In case a degradation of construction material is detected, the extent thereof is evaluated along with influence on the behaviour of the whole system and future development. The first two phases are inherently associated with non-destructive evaluation (NDE) of materials.

Different physical principles are utilised for the NDE of materials. Eddy current testing (ECT) is one of the widely utilized electromagnetic methods. It originates from the electromagnetic induction phenomena. The principle of ECT underlies in the interaction of induced eddy currents with structure of an examined body [1].

Commercial ECT systems provide raw data with limited or absent capability of their quantitative interpretation [2]. The progress in powerful computers has allowed developing of automated procedures to estimate dimensions of a detected anomaly; however, they are not commercially available yet. The stochastic or the deterministic methods are employed in the automated procedures for sizing of an indicated crack [3]. Usually, one dimensional signal gained by scanning just above the crack along its length is taken as an input to the evaluation procedure. Mostly three variables of the crack are estimated, i.e. a depth, a length and a position of its centre, while a profile, a width and the electromagnetic properties of the defect have to be adjusted in advance. Satisfactory results are reported by several groups for evaluation of artificial slits [4]. However, evaluation of real cracks, especially stress corrosion cracking (SCC) from ECT response signals remains still very difficult [4]. It has been found out that an SCC is partially conductive [5] while its conductivity is not known in general and can vary from one case to another case.

The paper deals with evaluation of uncertainty in depth estimation of a detected partially conductive crack.

\section{Principle of ECT}

The principle of the ECT underlies in the interaction of induced eddy currents with a structure of an examined body [1].

An alternating electromagnetic field is generated in the vicinity of a coil driven by a time-varying current. It can be simply considered as a superposition of a primary exciting field and a secondary one generated by eddy currents. The secondary electromagnetic field counterworks to the primary exciting electromagnetic field according to the Lenz's theorem. According to the Ampere's law:

\footnotetext{
* Ladislav Janousek, Milan Smetana

Department of Electromagnetic and Biomedical Engineering, Faculty of Electrical Engineering, University of Zilina, Slovakia,

E-mail: janousek@fel.uniza.sk
} 


$$
\begin{aligned}
& \nabla \times \mathbf{H}_{p}=\mathbf{J}_{e x}, \\
& \nabla \times \mathbf{H}_{e d}=\mathbf{J}_{e d}, \\
& \mathbf{H}=\mathbf{H}_{p}+\mathbf{H}_{e d},
\end{aligned}
$$

where $\mathbf{H}_{p}, \mathbf{H}_{e d}, \mathbf{H}\left[\right.$ A. $\mathrm{m}^{-1}$ ] denote the magnetic field intensity vector of the primary, secondary and resulting field, respectively, and $\mathbf{J}_{e x}, \mathbf{J}_{e d}\left[\mathrm{~A} \cdot \mathrm{m}^{-2}\right.$ ] are the current density vectors of the exciting current and of the eddy currents, respectively. Electromotive force is induced in a conductive object which is in proximity of the coil according to the Faraday's law:

$$
\nabla \times \mathbf{E}=-\frac{\partial \mathbf{B}}{\partial t},
$$

where $\mathbf{E}\left[\mathrm{V} \cdot \mathrm{m}^{-1}\right]$ is the electromotive force vector and $\mathbf{B}[\mathrm{T}]$ is the magnetic flux density vector, while $\mathbf{B}=\mu \mathbf{H}$, and $\mu\left[\mathrm{H}_{\mathrm{m}} \mathrm{m}^{-1}\right]$ is the magnetic permeability.

Eddy-currents flow in the conductive object according to the Ohm's law:

$$
\mathbf{J}_{e d}=\gamma \mathbf{E},
$$

where $\gamma\left[\mathrm{S}^{-1} \mathrm{~m}^{-1}\right]$ is the electric conductivity. Their vector lines must be closed due to:

$$
\nabla \cdot \mathbf{J}_{e d}=0
$$

The induction coupling therefore exists between the coil and the conductive object. The resulting electromagnetic field of the coil and the conductive object depends on geometrical parameters of the system as well as on the electromagnetic parameters of the conductive object.

The principle of the ECT has been known for several decades. Nowadays, the most wide spread application area of the ECT is the detection and possible evaluation of different discontinuities in conductive materials.

Presence of a defect in a conductive material causes a local change of the material electromagnetic parameters. As the eddy current vector lines must be encircled, the presence of a defect changes the eddy current density distribution and thus influences the resulting electromagnetic field. The perturbation electromagnetic field therefore occurs comparing to the no-crack situation and this perturbation field can be sensed and further evaluated. The ECT is a relative method and the perturbation signal is obtained by subtracting of the crack signal and no-crack signal. The perturbation signal carries quantitative information about an inspected defect.

The ECT posses several benefits:

- high sensitivity for surface breaking defects,

- high inspection speed,

- contact-less inspection,

- versatility, especially comparing to the ultrasonic testing, one of the most utilized non-destructive techniques. These advantages determine continuously enlarging application area of ECT particularly in nuclear, petrochemical and aviation industries.

On the other hand, also disadvantages of the method should be mentioned. The ECT signals are integral values and they do not carry explicit information about crack dimensions. It means that the inverse problem is ill-posed. Therefore, evaluating the depth of a defect from the ECT signals is quite difficult. In addition, the skin-effect concentrates induced currents on the surface of a tested material. Eddy current density decays almost exponentially into material depth and thus increasing depth of a surface breaking defect causes raising uncertainty of the depth evaluation because of the ECT signal saturation. The situation gets even worse when partially conductive cracks (i.e. SCCs) are expected and need to be detected and evaluated.

\section{Numerical Model}

A plate specimen having the electromagnetic parameters of a stainless steel SUS316L is inspected in this study. The specimen has a thickness of $t=10 \mathrm{~mm}$, a conductivity of $\sigma=1.4 \mathrm{MS} / \mathrm{m}$ and a relative permeability of $\mu_{r}=1$. A surface breaking crack appears in the middle of the plate. It is modelled as the cuboid having different electromagnetic properties from the base material. Configuration of the plate with the crack is shown in Fig. 1. A length of the crack is fixed to $l_{c}=10 \mathrm{~mm}$.

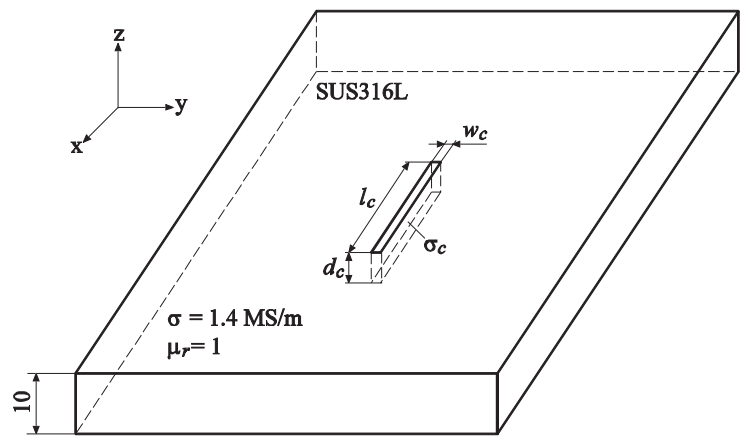

Fig. 1 Layout of specimen

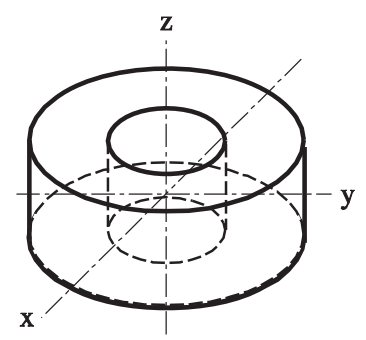

Fig. 2 Configuration of pancake coil probe 
A probe shown in Fig. 2 is employed to inspect the crack. This probe is known as the pancake one and it is widely used in practice. The probe is of self-inductance absolute type, i.e. one coil is the exciter and the detector. Outer diameter of the probe has a value of $3.0 \mathrm{~mm}$, its inner diameter is set to $1.0 \mathrm{~mm}$ and height of the winding is $1.0 \mathrm{~mm}$ in this case. The probe is driven with the harmonic current having a frequency of $10 \mathrm{kHz}$. The standard depth of penetration equals to $\delta=4.2 \mathrm{~mm}$ under the given conditions.

Response signals sensed by the probe are calculated by numerical means. One dimensional scanning along the crack length as shown in Fig. 3 is considered here. Such one dimensional signal is usually used by an automated evaluation procedure. Clearance between the plate surface and the probe, so called lift-off is adjusted to $1 \mathrm{~mm}$

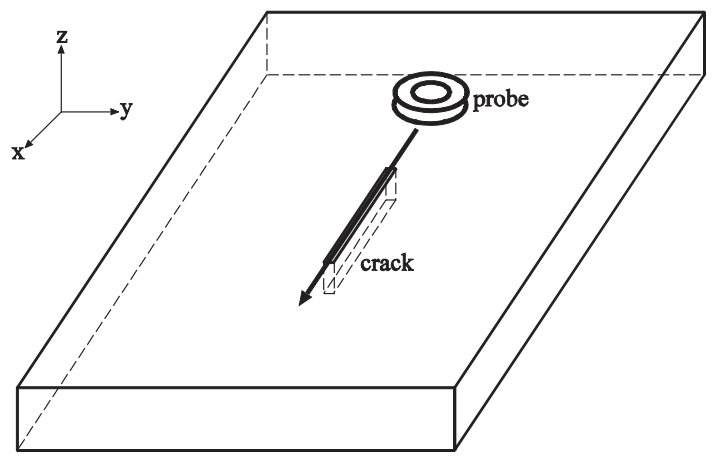

Fig. 3 One dimensional scanning pattern

Three parameters of the crack are varied, i.e. its depth $d_{c}$, width $w_{c}$ and conductivity $\sigma_{c}$ to simulate various structures of real conductive cracks. The depth is changed from 10 to $90 \%$ of the plate thickness with a step of $10 \%$. The width of crack $w_{c}$ is adjusted to five values ranging from $0.2 \mathrm{~mm}$ until $1.0 \mathrm{~mm}$ with a step of $0.2 \mathrm{~mm}$ Considered conductivities of the crack are as follows: 0 , $1,2,5$ and $10 \%$ of the base material conductivity. Influences of the crack parameters on the sensed ECT response signals are studied. The results are presented and discussed in the next section.

\section{Numerical Results and Discussion}

Numerical simulations are carried out to evaluate influence of the crack conductivity on uncertainty of the crack's depth estimation. A user developed edge-element code based on the finite element method is used for the calculations. The code calculates the real and the imaginary parts of the complex probe signal under the harmonic excitation according to the symbolic-complex method. The signal is calculated for each probe's position along the scanning line as shown in Fig. 3. The probe moves over the plate surface without crack to get a reference dependence of the signal on the probe position at first. The simulations are then repeated for the cracked scenarios under the same conditions. The dependencies of the crack response signals are calculated for each crack in such a way that the reference dependence is subtracted from the dependence gained under a cracked scenario. Following figures display the dependences of the absolute value of the complex cracks' response signals on the probe position relative to the crack centre as well as they show the complex cracks' response signals in the complex plane.

The response signals of the non-conductive crack with various depths are shown in Fig. 4. It can be seen that the crack signals start to saturate when the crack is deeper than approximately $70 \%$ of the plate thickness, i.e. $167 \%$ of the standard depth of penetration $\delta$. Similar results are displayed in Fig. 5. However, the crack is partially conductive in this case; the crack conductivity equals to $10 \%$ of the base material conductivity. It can be seen that the crack conductivity strongly influences saturation of the crack signals as in this case it is quite difficult to find differences between the signals when the crack is deeper than the standard depth of penetration $\delta$.

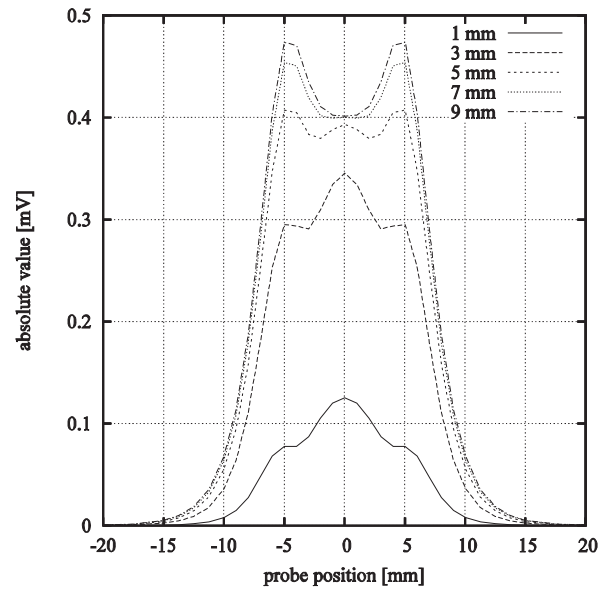

a) dependences of the absolute value on the probe position relative to the crack centre

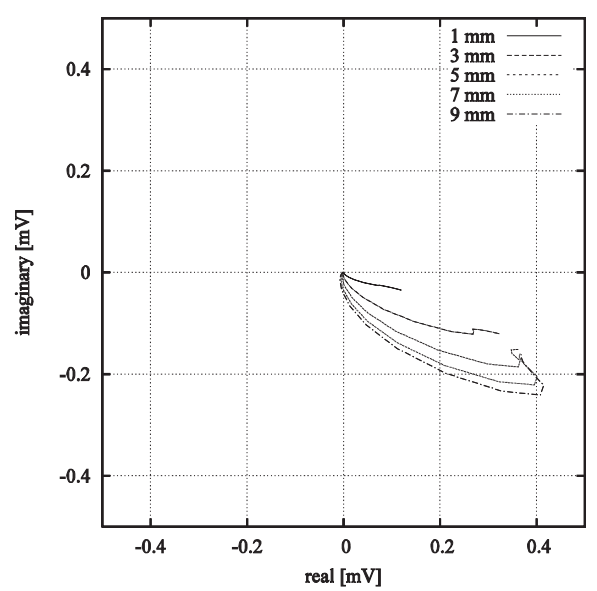

b) dependences of the imaginary part on the real part of the detected signal with respect to the phase of exciting voltage

Fig. 4 Signals of the non-conductive crack with a width of $w_{c}=0.2 \mathrm{~mm}$ and various depths, i.e. $d_{c}=1,3,5,7$ and $9 \mathrm{~mm}$ 


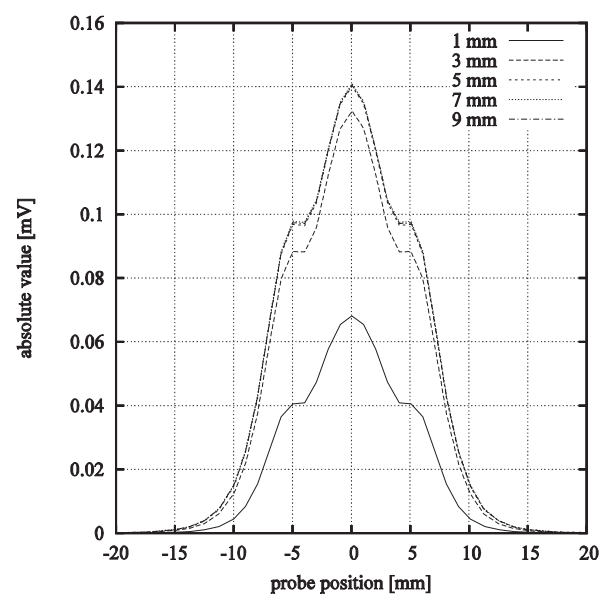

a) dependences of the absolute value on the probe position relative to the crack centre

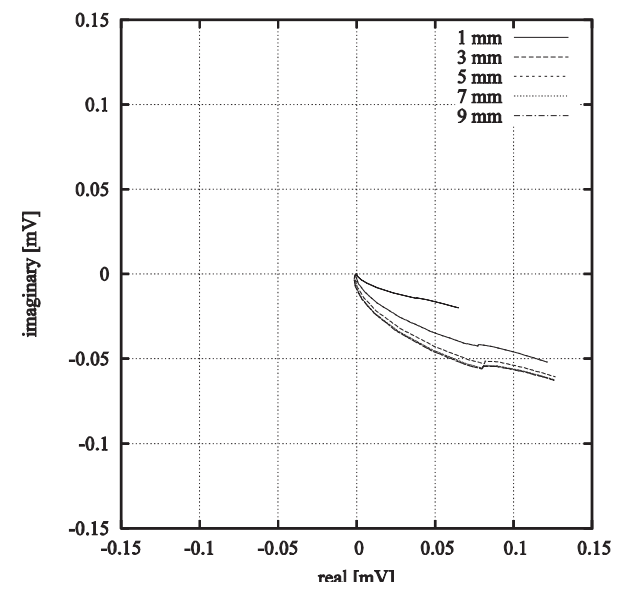

b) dependences of the imaginary part on the real part of the detected signal with respect to the phase of exciting voltage

Fig. 5 Signals of the crack with a width of $w_{c}=0.2 \mathrm{~mm}$, a partial conductivity of $\sigma_{c}=10 \%$ of the base material conductivity and various depths, i.e. $d_{c}=1,3,5,7$ and $9 \mathrm{~mm}$

Influence of the crack conductivity on the crack signals can be observed for the results presented in Fig. 6. The depth of crack is adjusted to $5 \mathrm{~mm}$ and its width is set to $0.2 \mathrm{~mm}$ in this case while the crack's conductivity is varied. The presented results clearly show that the crack's conductivity strongly influences the response signals, while a crack with higher partial conductivity provides the response signal with lower amplitude.

Several studies [4] have concluded that in case of conductive cracks also a width of the cracking significantly affects the measured responses.

Figs. 7 and 8 show the crack response signals for the crack with variable width, while the crack's depth is adjusted to $5 \mathrm{~mm}$. The crack's partial conductivity is set to $0 \%$ for the first case and to $10 \%$ of the base material conductivity for the second case. When the crack is non-conductive the response signals only slightly depend

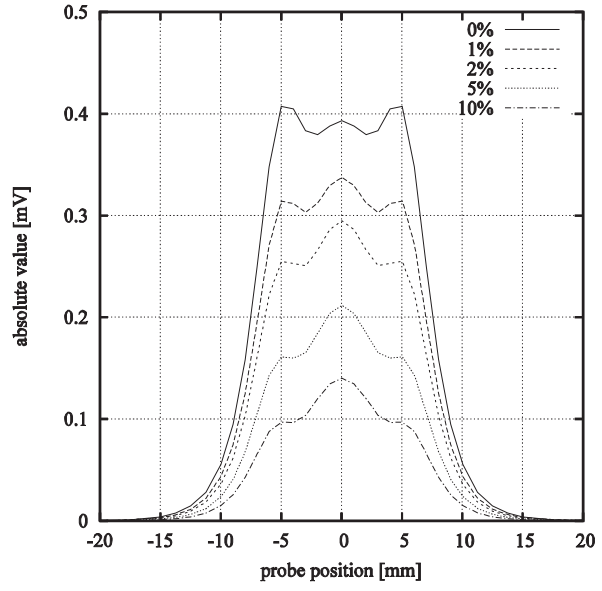

a) dependences of the absolute value on the probe position relative to the crack centre

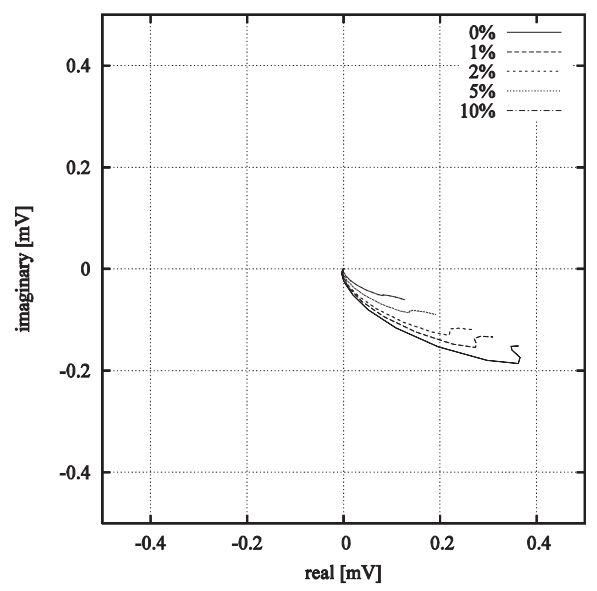

b) dependences of the imaginary part on the real part of the detected signal with respect to the phase of exciting voltage

Fig. 6 Signals of the crack with a depth of $d_{c}=5 \mathrm{~mm}$, a width of $w_{c}=0.2 \mathrm{~mm}$ and various values of the partial conductivity, i.e.

$\sigma_{c}=0,1,2,5$ and $10 \%$ of the base material conductivity

on the crack's width; however, when the crack is partially conductive the influence of the crack's width on the response signals is more significant.

Ambiguity of crack's depth estimation due to crack's conductivity is evaluated in a following way. Gained response signals of the crack with variable depth and conductivity are compared in such a way as the deterministic evaluation methods work. A difference between signals of two cracks denoted as $A$ and $B$ is calculated based on:

$$
\varepsilon_{A B}=\sum_{i=1}^{n} Z_{A, i}-Z_{B, i}{ }^{2},
$$

as proposed in [6]. $Z_{A, i}$ represents the response signal of a crack being considered as an inspected one at the $i$ th scanning point. 


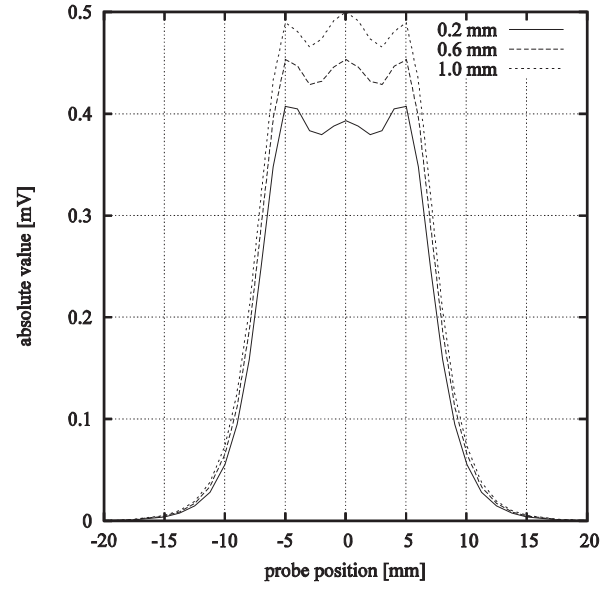

a) dependences of the absolute value on the probe position relative to the crack centre

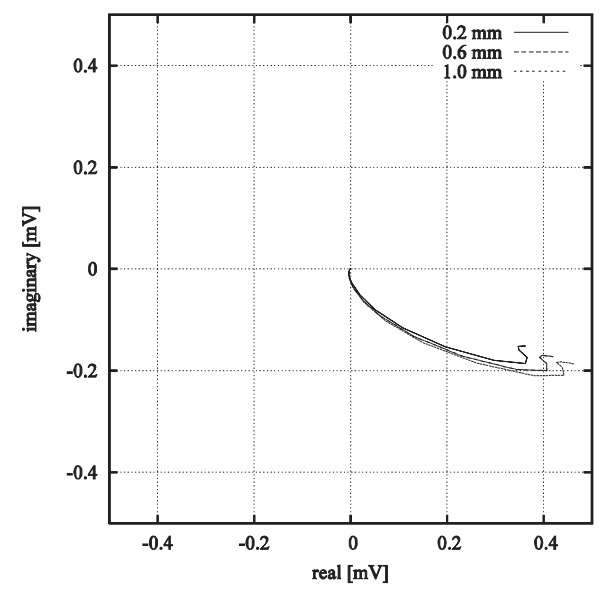

b) dependences of the imaginary part on the real part of the detected signal with respect to the phase of exciting voltage

Fig. 7 Signals of the crack with a depth of $d_{c}=5 \mathrm{~mm}$, a partial conductivity of $\sigma_{c}=0 \%$ of the base material conductivity and variable width, i.e. $w_{c}=0.2,0.6$ and $1.0 \mathrm{~mm}$

Thus a depth of this crack is taken as the true one. $Z_{B, i}$ represents the response signal of a crack being considered as a model of the inspected one at the $i$ th scanning point. Its depth is denoted as the estimated one. Total number of scanning points is $n$. Every possible combination of two cracks with different parameters is treated in this manner. If the calculated difference $\varepsilon_{A B}$ between the response signals of cracks $A$ and $B$ is lower or equal to $1 \%$, depth of the crack $A$ is estimated to be equal to that one of the crack $B$.

Fig. 9 shows relationship between the true and the estimated depths of the crack A. Only non-conductive cracks with different depths are considered in this case. The full line corresponds to the ideal case when the estimated depth equals to the true one. The relation between the true and the estimated depth according to the above defined criterion is represented by the stars. It can be seen that the uncertainty in the crack depth estimation increases when the crack gets deeper than the standard depth of penetration $\delta(40 \%$ of the plate thickness in this case) due to the signal saturation as mentioned earlier.

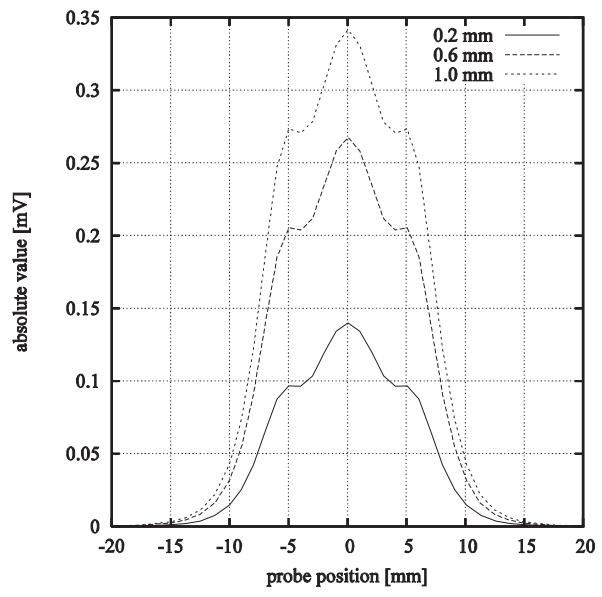

a) dependences of the absolute value on the probe position relative to the crack centre

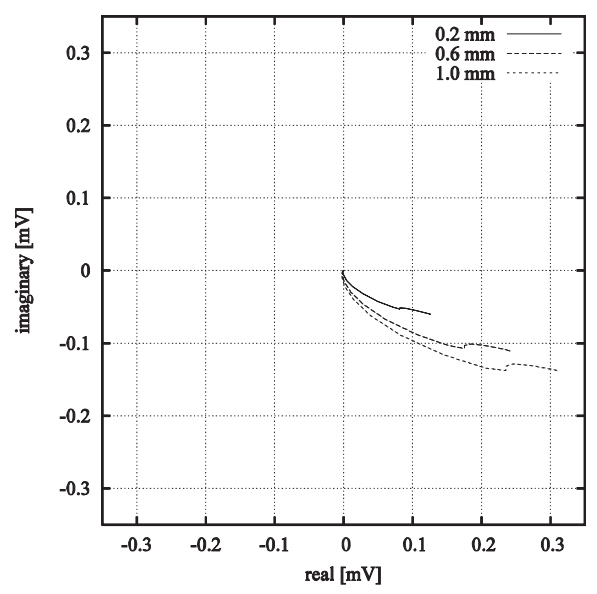

b) dependences of the imaginary part on the real part of the detected signal with respect to the phase of exciting voltage

Fig. 8 Signals of the crack with a depth of $d_{c}=5 \mathrm{~mm}$, a partial conductivity of $\sigma_{c}=10 \%$ of the base material conductivity and variable width, i.e. $w_{c}=0.2,0.6$ and $1.0 \mathrm{~mm}$

It means that the non-conductive crack with a depth of $90 \%$ is likely to be underestimated at maximum of approximately $30 \%$ of its true depth.

When the crack conductivity is taken into consideration the situation gets considerably worse as shown in Fig. 10. Even depth of shallow cracks can be highly underestimated.

It clearly comes from the presented results that one needs to be very careful when estimating depth of a conductive, i.e. stress corrosion cracks. 


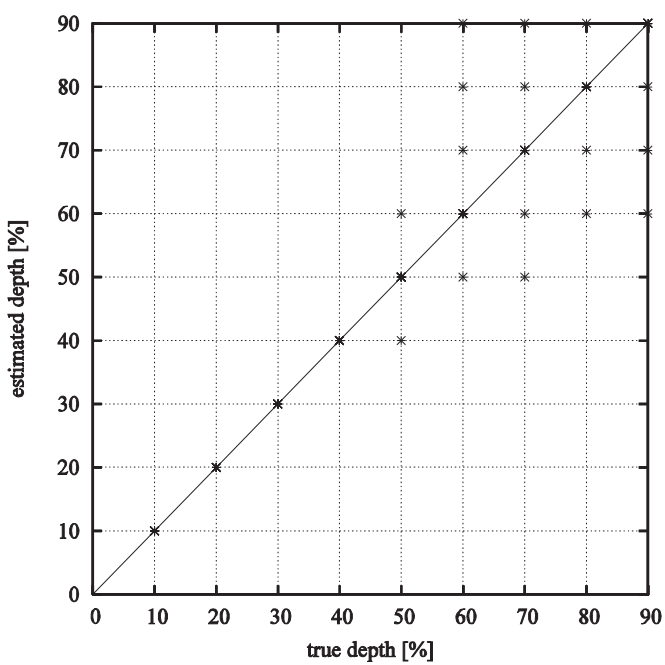

Fig. 9 Relationship between true and estimated depths of a non-conductive crack

\section{Conclusion}

The paper focused on depth estimation of partially conductive cracks from eddy current response signals. A plate specimen having electromagnetic characteristics of a stainless steel SUS316L was inspected in this study. Response signals of a crack with rectangular shape were calculated by numerical means for a standard pancake probe. Three parameters of the crack, its depth, width and partial conductivity were varied to simulate heterogeneity of real cracks. It was shown that partial conductivity of a crack strongly influences the response signal's saturation level as well as highlights influence of a crack width on the response signal. Thus the dimension of unknown parameters of a detected crack increases and the inverse problem gets highly ill-posed. The presented results evidently showed that ambiguity of dimensions' evaluation of an indicated

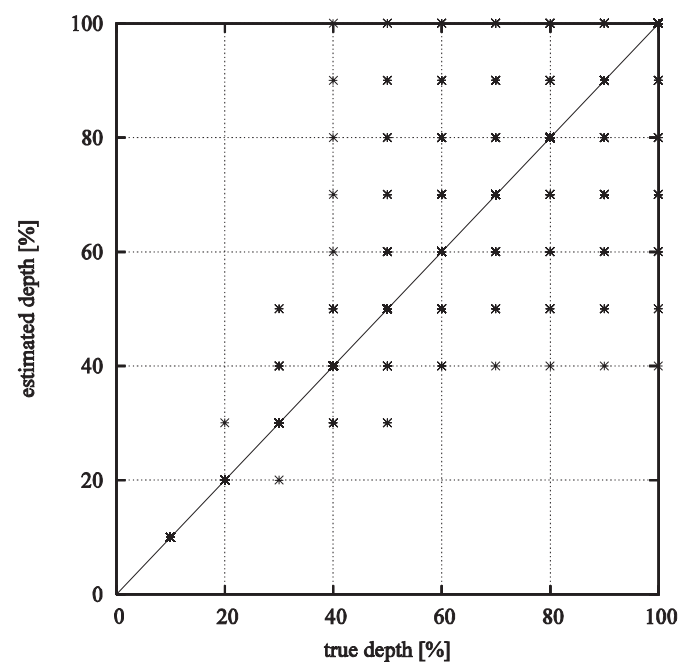

Fig. 10 Relationship between true and estimated depths of a conductive crack

crack considerably increases with crack's conductivity. Thus, special attention needs to be devoted to dimensions' evaluation of stress corrosion cracking where not all the ligaments are broken and the crack is partially conductive.

\section{Acknowledgement}

This work was supported by the Slovak Research and Development Agency under the contract No. APVV-0194-07. This work was also supported by grant of the Slovak Grant Agency VEGA, project No. $1 / 0765 / 11$.

The authors wish to thank for the support to the R\&D operational program Centre of excellence of power electronics systems and materials for their components, No. OPVaV-2008/2.1/01SORO, ITMS 26220120003 funded by European Community.

\section{References}

[1] JANOUSEK, L., MAREK, T., GOMBARSKA, D.: Eddy Current Non-destructive Evaluation of Conductive Materials, Communications - Scientific Letters of the University of Zilina, 1, pp. 29-33, 2006.

[2] RUBINACCI, G., TAMBURINO, A., VENTRE, S.: Fast Numerical Techniques for Electromagnetic Nondestructive Evaluation, Nondestructive Testing and Evaluation, 24, pp. 165-194, 2009.

[3] CHEN, Z., YUSA, N., MIYA, K.: Some Advances in Numerical Analysis Techniques for Quantitative Electromagnetic Nondestructive Evaluation, Nondestructive testing and evaluation, 24, pp. 69-102, 2009.

[4] YUSA, N.: Development of Computational Inversion Techniques to Size Cracks from Eddy Current Signals, Nondestructive Testing and Evaluation, 24, pp. 39-52, 2009.

[5] YUSA, N., MIYA, K.: Discussion on the Equivalent Conductivity and Resistance of Stress Corrosion Cracks in Eddy Current Simulations, NDT\&E International, 42, pp. 9-15, 2009.

[6] Yusa, N., Huang, H., Miya, K.: Numerical Evaluation of the Ill-posedness of Eddy Current Problems to Size Real Cracks, NDT\&E International, 40, pp. 185-191, 2007. 